\title{
Supersymmetric Standard Model and Radiative Meson Decay*
}

\author{
Noriyuki Oshimo ${ }^{a}$ \\ ${ }^{a}$ Grupo Teórico de Altas Energias, CFIF, \\ Instituto Superior Técnico, \\ Av. Rovisco Pais, 1096 Lisboa Codex, Portugal
}

I give a review on the generation-changing interactions of the supersymmetric standard model based on grand unification and $N=1$ supergravity. These interactions could make sizable effects on rare processes related to $B$-mesons. The decay $b \rightarrow s \gamma$ is discussed in particular. It is shown that the inclusive branching ratio of the radiative $B$-meson decay is deviated from the standard model prediction in nontrivial ranges for parameter values.

\section{INTRODUCTION}

The $B$-meson decay into a strange hadronic state and a photon, which is induced by the decay $b \rightarrow s \gamma$ at the quark level, is now one of the hot topics in the particle physics phenomenology. This b-quark decay process of flavor-changing neutral current (FCNC) is predicted by the standard electroweak theory through the one-loop diagrams in which the $W$-bosons and the up-type quarks are exchanged. Recently the CLEO collaboration has observed the decays $B^{0} \rightarrow K^{* 0} \gamma$ and $B^{-} \rightarrow K^{*-} \gamma$ with their average branching ratio $(4.5 \pm 1.5 \pm 0.9) \times 10^{-5}[1]$. These observations provide a big opportunity for sensitively examining the standard $\mathrm{SU}(3) \times \mathrm{SU}(2) \times \mathrm{U}(1)$ model.

In the theoretical study of the radiative $B$ meson decay there seems to be three aspects. First, QCD corrections are important for predicting the branching ratio of $b \rightarrow s \gamma$ within the framework of the standard model. There already appeared a number of articles [2] which discuss these corrections, concluding enhanced values for $\mathrm{BR}(b \rightarrow s \gamma)$ by a factor $2-5$ compared to the noncorrected one. Second, the evaluation of the relevant hadronic matrix elements are necessary to obtain the branching ratios of the exclusive $B$-meson decay modes. Theoretical estimates for the ratio of $\mathrm{BR}\left(B \rightarrow K^{*} \gamma\right)$ to $\mathrm{BR}(b \rightarrow s \gamma)$ are yet dispersed from 0.05 to 0.4 [3]. Third, the effects of the theories beyond the standard model may

\footnotetext{
* to appear in the Proceedings of the XVth Autumn School 'Particle Physics in the Nineties'.
}

be observed. In particular, the supersymmetric models $[4,5,6]$ and the two Higgs-doublet models [7] could sizably contribute to the decay $b \rightarrow s \gamma$.

In this lecture we discuss the third aspect, concentrating on the effects of the supersymmetric standard model (SSM). In this model FCNC processes can be induced by several new sources as well as the standard mechanism mediated by the $W$-bosons. These are the interactions in which the quark $q$ couples to the squark $\tilde{q}$ and the chargino $\tilde{\omega}$, the neutralino $\tilde{\chi}$, or the gluino $\tilde{g}$, the quark and the squark belonging to different generations. As a general feature of those models which contain two doublets of Higgs bosons, the charged Higgs bosons $H^{ \pm}$also mediate FCNC processes. Therefore, various one-loop diagrams have to be appropriately taken into consideration to obtain the SSM prediction. For definiteness, we assume grand unification theories coupled to $N=1$ supergravity. It is considered that FCNC processes in the $B$-meson system, in general, could play an important role in studying the SSM. The scheme describing the generationchanging interactions given in this lecture would be useful also for discussing other $B$-meson rare phenomena.

In sect. 2 we derive the interaction lagrangians of the SSM which incorporate the generation mixings for the quarks and the squarks. In sect. 3 formulae are given for the radiative decay width of a quark. In sect. 4 we make numerical analyses on the branching ratio of the inclusive radiative $B$-meson decay. The summary is given in sect. 5 . 


\section{INTERACTIONS}

We start from describing the interactions which induce generation-changing processes. The lagrangian of the SSM is written in matrix form

$$
\begin{aligned}
& L=L_{k i n}+L_{\text {gauge }}+L_{F}+L_{S} ; \\
& L_{F}=\left[H_{1} \times L \eta_{E} E^{c}\right. \\
& +H_{1} \times Q \eta_{D} D^{c}+H_{2} \times Q \eta_{U} U^{c} \\
& \left.+m_{H} H_{1} \times H_{2}\right]_{F}+\text { h.c. } \\
& L_{S}=-m_{3 / 2}\left(\tilde{H}_{1} \times \tilde{L} A_{E} \eta_{E} \tilde{E}^{c}\right. \\
& +\tilde{H}_{1} \times \tilde{Q} A_{D} \eta_{D} \tilde{D}^{c}+\tilde{H}_{2} \times \tilde{Q} A_{U} \eta_{U} \tilde{U^{c}} \\
& +B m_{H} \tilde{H}_{1} \times \tilde{H}_{2} \text { ) } \\
& -\sum_{i=1}^{3} \tilde{m}_{i} \operatorname{Tr}\left(T_{i}^{a} T_{i}^{b}\right) \lambda_{i}^{a} \frac{1-\gamma_{5}}{2} \lambda_{i}^{b}+\text { h.c. } \\
& -\tilde{L}^{\dagger} \tilde{M}_{L}^{2} \tilde{L}-\tilde{E}^{c}{ }^{\dagger} \tilde{M}_{E}^{2} \bar{E}^{c} \\
& -\tilde{Q}^{\dagger} \tilde{M}_{Q}^{2} \tilde{Q}-\tilde{D}^{c}{ }^{\dagger} \tilde{M}_{\bar{D}}^{2} \tilde{D}^{c}-\tilde{U}{ }^{\dagger} \tilde{M}_{U}^{2} \tilde{U}^{c} \\
& -\tilde{M_{H 1}^{2}} \tilde{H}_{1}^{\dagger} \tilde{H}_{1}-\tilde{M_{H 2}^{2}} \tilde{H}_{2}^{\dagger} \tilde{H}_{2},
\end{aligned}
$$

where $L$ etc. represent superfields: $\tilde{L}$, etc. their scalar components; and $\lambda_{1}, \lambda_{2}$, and $\lambda_{3}$ are respectively $U(1), S U(2)$, and $S U(3)$ gauginos. The generators of the gauge groups are denoted by $T_{i}$. For simplicity we assume that the particle content is minimal. Without loss of generality the gravitino mass $m_{3 / 2}$, the gaugino masses $\tilde{m}_{i}$, and the vacuum expectation values $v_{1}$ and $v_{2}$ of the Higgs bosons $\tilde{H}_{1}$ and $\tilde{H}_{2}$ can be taken as real and non-negative. Other parameters are generally complex. The terms in $L_{S}$ break supersymmetry softly.

The quark fields and the squark fields are respectively mixed in the generation space. Since the left-handed squarks and the right-handed ones are also mixed, the mass-squared matrices for the up-type and down-type squarks, $M_{U}^{2}$ and $M_{D}^{2}$, are expressed by $6 \times 6$ matrices as

$$
\begin{aligned}
M_{F}^{2}= & \left(\begin{array}{ll}
M_{F 11}^{2} & M_{F 12}^{2} \\
M_{F 21}^{2} & M_{F 22}^{2}
\end{array}\right) ; \\
M_{F 11}^{2}= & \cos 2 \beta\left(T_{3 F}-Q_{F} \sin ^{2} \theta_{W}\right) M_{Z}^{2} E \\
& +m_{F} m_{F}^{\dagger}+\tilde{M}_{Q}^{2}, \\
M_{F 22}^{2}= & \cos 2 \beta Q_{F} \sin ^{2} \theta_{W} M_{Z}^{2} E
\end{aligned}
$$

$$
\begin{aligned}
& +m_{F}^{\dagger} m_{F}+\tilde{M}_{F}^{2} \\
M_{F 12}^{2}= & M_{F 21}^{2 \dagger}=R_{F} m_{H} m_{F}+m_{3 / 2} A_{F}^{*} m_{F}, \\
& (F=U, D) .
\end{aligned}
$$

Here $m_{U}$ and $m_{D}$ are the mass matrices of the up-type and down-type quarks; $R_{U}=1 / \tan \beta$ and $R_{D}=\tan \beta, \tan \beta$ being $v_{2} / v_{1}$; and $E$ is the $3 \times 3$ unit matrix. The quark mass matrices are diagonalized by unitary matrices as

$$
\begin{aligned}
U_{L}^{U} m_{U} U_{R}^{U^{\dagger}} & =\operatorname{diag}\left(m_{u}, m_{e}, m_{t}\right)\left(\equiv \overline{m_{U}}\right), \\
U_{L}^{D} m_{D} U_{R}^{D^{\dagger}} & =\operatorname{diag}\left(m_{d}, m_{s}, m_{b}\right)\left(\equiv \overline{m_{D}}\right) .
\end{aligned}
$$

The Cabbibo-Kobayashi-Maskawa (CKM) matrix $V$ is equivalent to $U_{L}^{U} U_{L}^{D} \dagger$.

The squark mass eigenstates are obtained by diagonalizing $M_{U}^{2}$ and $M_{D}^{2}$, which contain unknown matrices $\tilde{M}_{Q}^{2}, \tilde{M}_{U}^{2}, \tilde{M}_{D}^{2}, A_{U}$, and $A_{D}$ coming from the soft-breaking lagrangian $L_{S}$ in eq. (1). One possible way of study is to discuss constraints on the squark mass matrices from a purely phenomenological view without assuming a priori any specific forms for them. Here, instead, we make certain assumptions suggested by the supergravity models.

At the very high energy where supersymmetry is broken, it is expected that $\tilde{M}_{Q}^{2}=\tilde{M}_{U}^{2}=\tilde{M}_{D}^{2}=$ $m_{3 / 2}^{2} E$ and $A_{U}=A_{D}=a E, a$ being a number of order unity. At the lower energy scale, quantum effects give corrections to these relations [8]. The masses of the left-handed squarks $\tilde{Q}$ are induced by both of the two types of Yukawa interactions proportional to $m_{U}$ and $m_{D}$. In the former case the up-type and down-type components of $\tilde{Q}$ get masses through loop diagrams with the neutral and the charged Higgs particles, respectively while in the latter case through loop diagrams with the charged and the neutral Higgs particles, respectively. The masses of the right-handed squarks $\tilde{U}^{c}$ and $\tilde{D}^{c}$ are induced, respectively, by the Yukawa interactions proportional to $m_{U}$ and $m_{D}$. Both the loop diagrams with the neutral Higgs particles and those with the charged Higgs ones contribute to their masses. The squarks also get masses through the gauge interactions, which do not depend on their generations. These con- 
tributions lead to the soft-breaking mass terms at the electroweak scale given roughly by

$$
\begin{aligned}
\tilde{M}_{Q}^{2} & =\tilde{m}_{Q}^{2} E+c m_{U} m_{U}^{\dagger}+d m_{D} m_{D}^{\dagger}, \\
\tilde{M}_{U}^{T} & =\tilde{m}_{U}^{2} E+2 c m_{U}^{\dagger} m_{U}, \\
\tilde{M}_{D}^{T} & =\tilde{m}_{D}^{2} E+2 d m_{D}^{\dagger} m_{D},
\end{aligned}
$$

where the coefficients $c$ and $d$ are negative and of order unity. On the other hand, $A_{U}^{*} m_{U}$ and $A_{D}^{*} m_{D}$ remain roughly proportional to $m_{U}$ and $m_{D}$, respectively.

Neglecting small contributions from the quark masses, then, the squark mixings among different generations are removed by the $6 \times 6$ unitary matrices

$$
\left(\begin{array}{cc}
U_{L}^{U} & 0 \\
0 & U_{R}^{U}
\end{array}\right) \cdot\left(\begin{array}{cc}
U_{L}^{U} & 0 \\
0 & U_{R}^{D}
\end{array}\right)
$$

for the up-type and the down-type squarks, respectively. The mixings of the left- and righthanded squarks are not neglected for the $t$ squarks and the $b$-squarks. The $2 \times 2$ masssquared matrices for the $t$ - and $b$-squarks, $M_{t}^{2}$ and $M_{b}^{2}$, are given by

$$
\begin{aligned}
M_{t 11}^{2}= & \cos 2 \beta\left(\frac{1}{2}-\frac{2}{3} \sin ^{2} \theta_{W}\right) M_{Z}^{2} \\
& +(1-|c|) m_{t}^{2}+\tilde{m_{Q}}{ }^{2}, \\
M_{t 22}^{2}= & \frac{2}{3} \cos 2 \beta \sin ^{2} \theta_{W} M_{Z}^{2} \\
& +(1-2|c|) m_{t}^{2}+\tilde{m_{U}}{ }^{2}, \\
M_{t 12}^{2}= & M_{t 21}^{2^{*}}=\left(\frac{1}{\tan \beta} m_{H}+a_{t}^{*} m_{3 / 2}\right) m_{t}, \\
M_{b 11}^{2}= & \cos 2 \beta\left(-\frac{1}{2}+\frac{1}{3} \sin ^{2} \theta_{W}\right) M_{Z}^{2} \\
& -|c| m_{t}^{2}+\tilde{m_{Q}}{ }^{2}, \\
M_{b 22}^{2}= & -\frac{1}{3} \cos 2 \beta \sin ^{2} \theta_{W} M_{Z}^{2}+\tilde{m_{D}}{ }^{2}, \\
M_{b 12}^{2}= & M_{b 21}^{2^{*}}=\left(\tan \beta m_{H}+a_{b}^{*} m_{3 / 2}\right) m_{b} .
\end{aligned}
$$

These are readily diagonalized by $2 \times 2$ unitary matrices, which are denoted by $S_{t}$ and $S_{b}$. We express the mass eigenstates of the up-type and down-type squarks by $\left(\tilde{u_{L}}, \tilde{c_{L}}, \tilde{t_{1}}, \tilde{u_{R}}, \tilde{c_{R}}, \tilde{t_{2}}\right)$ and $\left(\tilde{d_{L}}, \tilde{s_{L}}, \tilde{b_{1}}, \tilde{d_{R}}, \tilde{s_{R}}, \tilde{b_{2}}\right)$. In our approximation the squarks in the second generation have the same masses as their counterparts in the first generation have.

The charginos and neutralinos are the charged and neutral mass eigenstates of the $\mathrm{SU}(2) \times \mathrm{U}(1)$ gauginos and the higgsinos. The mass matrices for the charginos and the neutralinos are given respectively by

$M^{-}=\left(\begin{array}{cc}\tilde{m}_{2} & -g v_{1} / \sqrt{2} \\ -g v_{2} / \sqrt{2} & m_{H}\end{array}\right)$,

$$
\begin{aligned}
& M^{0} \\
& =\left(\begin{array}{cccc}
\tilde{m}_{1} & 0 & g^{\prime} v_{1} / 2 & -g^{\prime} v_{2} / 2 \\
0 & \tilde{m_{2}} & -g v_{1} / 2 & g v_{2} / 2 \\
g^{\prime} v_{1} / 2 & -g v_{1} / 2 & 0 & -m_{H} \\
-g^{\prime} v_{2} / 2 & g v_{2} / 2 & -m_{H} & 0
\end{array}\right)
\end{aligned}
$$

which are diagonalized by unitary matrices as

$$
\begin{aligned}
C_{R}^{\dagger} M^{-} C_{L} & =\operatorname{diag}\left(\tilde{m_{\omega 1}}, \tilde{m_{\omega 2}}\right), \\
N^{t} M^{0} N & =\operatorname{diag}\left(\tilde{m_{\chi 1}}, \tilde{m_{\chi 2}}, \tilde{m_{\chi^{3}}}, \tilde{m_{\chi^{4}}}\right) .
\end{aligned}
$$

The gluinos are equal to the $\mathrm{SU}(3)$ gauginos and their mass is given by $\tilde{m}_{g}=\tilde{m_{3}}$.

We can now write the couplings of the quarks to the squarks and the charginos, neutralinos, or gluinos in terms of the particle mass eigenstates. The interaction lagrangians for the downtype quarks and the charginos or gluinos incorporating the generation mixings are given by the following equations [5].

The chargino-quark-squark interaction:

$$
\begin{aligned}
& L_{\tilde{\omega}}=i \frac{g}{\sqrt{2}} \sum_{i=1}^{2}\left[\left({\tilde{u_{L}}}^{\dagger},{\tilde{c_{L}}}^{\dagger}, \sum_{k=1}^{2} S_{t_{k 1} \tilde{t}_{k}}^{\dagger}\right) V\right. \\
& \text {. } \overline{\tilde{\omega}}_{i}\left\{\sqrt{2} C_{R}^{*}{ }_{1 i}^{*}\left(\frac{1-\gamma_{5}}{2}\right)\right. \\
& \left.+\frac{m_{D}}{M_{W}} \frac{C_{L}^{*}}{\cos \beta}\left(\frac{1+\gamma_{5}}{2}\right)\right\}\left(\begin{array}{l}
d \\
s \\
b
\end{array}\right) \\
& -\frac{C_{R}^{*}{ }_{2 i}^{*}}{\sin \beta}\left(\tilde{u_{R}}{ }^{\dagger},{\tilde{c_{R}}}^{\dagger}, \sum_{k=1}^{2} S_{t k 2}{\tilde{t_{k}}}^{\dagger}\right) \frac{m_{U}}{M_{W}} V \\
& \left.\overline{\tilde{\omega}}_{i}\left(\frac{1-\gamma_{5}}{2}\right)\left(\begin{array}{l}
d \\
s \\
b
\end{array}\right)\right]+ \text { h.c. }
\end{aligned}
$$

In our approximation $\overline{m_{U}}$ and $\overrightarrow{m_{D}}$ should be taken as $\operatorname{diag}\left(0,0, m_{\imath}\right)$ and $\operatorname{diag}\left(0,0, m_{b}\right)$, leading to vanishing couplings for $\tilde{u_{R}}$ and $\tilde{c_{R}}$. 
The gluino-quark-squark interaction:

$$
\begin{aligned}
& L_{\tilde{g}}=i \sqrt{2} g_{3} \sum_{a=1}^{8}\left[\left({\tilde{d_{L}}}^{\dagger},{\tilde{s_{L}}}^{\dagger}, \sum_{k=1}^{2} S_{b k 1} \tilde{b}_{k}^{\dagger}\right) V\right. \\
& T_{3}^{a} \overline{\tilde{g^{a}}}\left(\frac{1-\gamma_{5}}{2}\right)\left(\begin{array}{l}
d \\
s \\
b
\end{array}\right) \\
& +\left(\tilde{d_{R}}, \tilde{s_{R}} \tilde{j}^{\dagger}, \sum_{k=1}^{2} S_{b_{k 2} \tilde{b}_{k}}^{\dagger}\right) \\
& \left.\cdot T_{3}^{a} \overline{g^{a}}\left(\frac{1+\gamma_{5}}{2}\right)\left(\begin{array}{l}
d \\
s \\
b
\end{array}\right)\right]+ \text { h.c.. }
\end{aligned}
$$

The neutralino-quark-squark interaction is also derived similarly.

\section{DECAY}

The generation-changing interactions yield the radiative decays of the quarks. The effective lagrangian for such a decay of a quark, $f_{1} \rightarrow f_{2} \gamma$, is generally written in terms of the form factors $F_{i}\left(q^{2}\right)$ and $G_{i}\left(q^{2}\right)$ as

$$
\begin{aligned}
L_{e f f}= & e \bar{f}_{2} \Gamma_{\mu} f_{1} A^{\mu} \\
\Gamma_{\mu}= & \left\{F_{1}\left(q^{2}\right)+G_{1}\left(q^{2}\right) \gamma_{5}\right\} \gamma_{\mu} \\
& +\left\{F_{2}\left(q^{2}\right)+G_{2}\left(q^{2}\right) \gamma_{5}\right\} \frac{i}{m_{1}+m_{2}} \sigma_{\mu \nu} q^{\nu} \\
& +\left\{F_{3}\left(q^{2}\right)+G_{3}\left(q^{2}\right) \gamma_{5}\right\} q_{\mu}, \\
& \left(q_{\mu}=p_{1 \mu}-p_{2 \mu}\right)
\end{aligned}
$$

where $p_{1}$ and $p_{2}$ stand for the momenta of the initial and final quarks. However. since $q^{2}=0$ and $q \cdot \epsilon=0$ are satisfied, $\epsilon$ being the polarization vector of the photon, the form factors $F_{3}(0)$ and $G_{3}(0)$ do not contribute to the decay amplitude. Also from the conservation of the electromagnetic current, $\left(m_{1}-m_{2}\right) F_{1}\left(q^{2}\right)+F_{3}\left(q^{2}\right) q^{2}=0$ and $\left(m_{1}+m_{2}\right) G_{1}\left(q^{2}\right)+G_{3}\left(q^{2}\right) q^{2}=0$ hold, so that $F_{1}(0)=G_{1}(0)=0$. Therefore only the dipole form factors $F_{2}(0)$ and $G_{2}(0)$ are relevant to the radiative decay. At the rest frame of the initial quark the decay width is given by

$$
\begin{aligned}
\Gamma\left(f_{1}-f_{2} \gamma\right)= & \frac{\alpha_{E M}}{2} m_{1}\left(\left|F_{2}(0)\right|^{2}+\left|G_{2}(0)\right|^{2}\right) \\
& \cdot\left(1-\frac{m_{2}}{m_{1}}\right)^{2}\left(1-\frac{m_{2}^{2}}{m_{1}^{2}}\right) .
\end{aligned}
$$

In the SSM the radiative quark decay $f_{1} \rightarrow$ $f_{2} \gamma$ is induced, except for the standard $W$-boson interactions, through the interactions of $f_{1}$ and $f_{2}$ with a spin- $\frac{1}{2}$ fermion $\psi$ and a spin- 0 boson $\phi$. In general, the interaction lagrangian for these particles is represented by

$$
\begin{aligned}
& L_{I}=g \bar{\psi}\left\{G_{L}^{1}\left(\frac{1-\gamma_{5}}{2}\right)+G_{R}^{1}\left(\frac{1+\gamma_{5}}{2}\right)\right\} f_{1} \phi^{\dagger} \\
& +g \bar{f}_{2}\left\{G_{L}^{2 *}\left(\frac{1+\gamma_{5}}{2}\right)+G_{R}^{2 *}\left(\frac{1-\gamma_{5}}{2}\right)\right\} \psi \phi .
\end{aligned}
$$

The dipole form factors receive contributions at the one-loop level. Let $m_{i}, M_{\psi}, M_{\phi}$ denote the masses of $f_{i}, \psi, \phi$ and $Q_{\psi}, Q_{\phi}$ denote the electric charges of $\psi, \phi$. Neglecting $\left(m_{i} / M_{\phi}\right)^{2}$ in the loop integrals, we get

$$
\begin{aligned}
F_{2}(0)= & \frac{g^{2}}{32 \pi^{2}} \frac{m_{1}+m_{2}}{M_{\phi}} \\
& {\left[\left(G_{L}^{2 *} G_{L}^{1}+G_{R}^{2 *} G_{R}^{1}\right) \frac{m_{1}+m_{2}}{M_{\phi}}\right.} \\
& \cdot\left\{-Q_{\psi} I_{1}(r)+Q_{\diamond} J_{1}(r)\right\} \\
& +\left(G_{R}^{2 *} G_{L}^{1}+G_{L}^{2 *} G_{R}^{1}\right) \frac{M_{\psi}}{M_{\phi}} \\
& \left.\cdot\left\{-Q_{\psi} I_{2}(r)+Q_{\odot} J_{2}(r)\right\}\right], \\
G_{2}(0)= & \frac{g^{2}}{32 \pi^{2}} \frac{m_{1}+m_{2}}{M_{\phi}} \\
& {\left[\left(G_{L}^{2 *} G_{L}^{1}-G_{R}^{2 *} G_{R}^{1}\right) \frac{m_{1}-m_{2}}{M_{\phi}}\right.} \\
& \cdot\left\{-Q_{\psi} I_{1}(r)+Q_{\odot} J_{1}(r)\right\} \\
- & \left(G_{R}^{2 *} G_{L}^{1}-G_{L}^{2 *} G_{R}^{1}\right) \frac{M_{\psi}}{M_{\phi}} \\
& \left.\cdot\left\{-Q_{\psi} I_{2}(r)+Q_{\odot} J_{2}(r)\right\}\right], \\
r & =\frac{M_{L}^{2}}{M_{\phi}^{2}},
\end{aligned}
$$

where the functions $I_{i}(r)$ and $J_{i}(r)$ are given by

$$
\begin{aligned}
I_{1}(r)= & \frac{1}{12(1-r)^{4}}\left(2+3 r-6 r^{2}+r^{3}\right. \\
& +6 r \ln r) \quad\left(I_{1}(1)=\frac{1}{24}\right), \\
I_{2}(r)= & \frac{1}{2(1-r)^{3}}\left(-3+4 r-r^{2}-2 \ln r\right) \\
& \left(I_{2}(1)=\frac{1}{3}\right),
\end{aligned}
$$




$$
\begin{aligned}
J_{1}(r)= & \frac{1}{12(1-r)^{4}}\left(1-6 r+3 r^{2}+2 r^{3}\right. \\
& \left.-6 r^{2} \ln r\right) \quad\left(J_{1}(1)=\frac{1}{24}\right), \\
J_{2}(r)= & \frac{1}{2(1-r)^{3}}\left(1-r^{2}+2 r \ln r\right) \\
& \left(J_{2}(1)=\frac{1}{6}\right) .
\end{aligned}
$$

The specific forms for the dipole form factors due to the interactions (12) and (13) are found in ref. [5].

\section{ANALYSES}

The radiative decay $b \rightarrow s \gamma$ is observed as the $B$-meson decay into a hadronic state involving a strange particle and a hard photon. One of the dominant decay modes for the $b$-quark is the three body decay into the $c$-quark, the electron, and the neutrino. Assuming that the $B$-meson decays are described by the $b$-quark decays, we can calculate the branching ratio for the inclusive radiative decay $B \rightarrow X_{s} \gamma$ by

$$
\begin{aligned}
& \operatorname{BR}\left(B \rightarrow X_{s} \gamma\right) \\
& \quad=\frac{\Gamma(b \rightarrow s \gamma)}{\Gamma\left(b \rightarrow c e \overline{\nu_{e}}\right)} \operatorname{BR}\left(B \rightarrow X_{c} e \overline{\nu_{e}}\right),
\end{aligned}
$$

where $\operatorname{BR}\left(B \rightarrow X_{c} e \overline{\nu_{e}}\right)$ is known experimentally to be 0.11 .

The decay width of $b \rightarrow s \gamma$ depends on several supersymmetric parameters. Assuming the relation between the gauge fine structure constants $\alpha_{i}$ and the gaugino masses $\tilde{m}_{i}$,

$\frac{\alpha_{3}}{\tilde{m_{3}}}=\frac{\alpha_{2}}{\tilde{m}_{2}}=\frac{5 \alpha_{1}}{3 \tilde{m}_{1}}$,

suggested by the grand unification theories, the masses and couplings of the charginos, neutralinos, and gluinos are determined by $\tan \beta, \tilde{m}_{2}$, and $m_{H}$. In the ordinary scheme for the gaugino mass generation, $\tilde{m}_{2}$ is smaller than or around the gravitino mass $m_{3 / 2}$. If the $S U(2) \times U(1)$ symmetry is broken through radiative corrections, $\tan \beta$ is most likely larger than unity and the magnitude of the higgsino mass parameter $m_{H}$ is at most of order $m_{3 / 2}$. The masses and couplings of the squarks are determined through many parameters, as seen in eqs. (6) and (7). The mass parameters $\tilde{m_{Q}}, \tilde{m_{U}}$, and $\tilde{m_{D}}$ are related to the gravitino mass and the gaugino masses. Their differences are considered to arise only from the electroweak interactions, which would be small compared to their values themselves, the scale of the squark masses. Therefore we assume approximately

$\tilde{m_{Q}}=\tilde{m_{U}}=\tilde{m_{D}}$.

The coefficients $a_{t}, a_{b}$, and $c$ depend on the supersymmetric parameters, though their magnitudes are of order unity. We simply take them as parameters and put

$c=0.5, \quad a_{t} m_{3 / 2}=a_{b} m_{3 / 2}=\tilde{m_{Q}}$.

The CKM matrix elements appearing in our calculations are $V_{23}, V_{32}$, and $V_{33}$. The one-loop diagrams with the squarks of the $i$-th generation lead to the term proportional to $V_{i 2}^{*} V_{i 3}$ for the decay amplitude. Since the squarks belonging to the first two generations have the same mass, their contributions are summed up to give the term proportional to $V_{32}^{*} V_{33}$ by the unitarity of the CKM matrix. We take $\left|V_{23}\right|=\left|V_{32}\right|$ and $\left|V_{33}\right|=1$, which are known to be in good approximation. No further evaluation is necessary for the CKM matrix. The mass of the $t$-quark is set for $150 \mathrm{GeV}$ as a typical example throughout our numerical analyses. We neglect QCD corrections, which does not change much the results.

Apart from the $W$-boson and charged Higgs boson contributions, the decay $b \rightarrow s \gamma$ is induced through the one-loop diagrams in which (a)the charginos and the up-type squarks, (b)the neutralinos and the down-type squarks, and (c)the gluinos and the down-type squarks are exchanged. One might think, and it was really assumed before, that among these three decay processes the process (c) makes a dominant contribution owing to the strong interaction. However, this is not the case. The contribution of (a) is larger than those of (b) and (c) in wide ranges of the parameter space [5].

The form factor receives contributions from the processes (a) and (c), respectively, roughly given by

$F_{2}^{\tilde{\omega}}(0) \sim \frac{\alpha_{2}}{8 \pi} V_{32}^{*} \frac{m_{b}^{2}}{\tilde{m}_{\omega} M_{W} \cos \beta}$ 


$$
\begin{gathered}
\cdot \frac{\tilde{m}_{\omega}{ }^{2}}{\tilde{m}_{t}{ }^{2}} I_{2}\left(\frac{\tilde{m_{\omega}}}{\tilde{m}_{t}{ }^{2}}\right), \\
F_{2}^{\tilde{g}}(0) \sim \frac{\alpha_{3}}{9 \pi} V_{32}^{*} \frac{m_{b}^{2}}{\tilde{m}_{g} \tilde{m}_{b}} \frac{\tilde{m}_{g}{ }^{2}}{\tilde{m}_{b}{ }^{2}} J_{2}\left(\frac{\tilde{m} g_{g}{ }^{2}}{\tilde{m}_{b}{ }^{2}}\right),
\end{gathered}
$$

where $1.4 \times 10^{-1}<r I_{2}(r)<5.0 \times 10^{-1}$ and $3.6 \times 10^{-2}<r J_{2}(r)<4.7 \times 10^{-1}$ for $0.1<r<$ 100. The factor $m_{b} / M_{W} \cos \beta$ in eq. (23) comes from the Yukawa couplings and $m_{b} / \tilde{m}_{b}$ in eq. (24) from the $\tilde{b}_{L}-\tilde{b}_{R}$ mixings. The gluino and chargino masses are given typically by $\tilde{m}_{3}$ and $\tilde{m}_{2}$, respectively. From eq. (20), therefore, the enhancement due to a larger value of $\alpha_{3}$ is weakened by a proportionally larger mass of the gluinos. We can see that $\left|F_{2}^{\tilde{\omega}}(0)\right|>\left|F_{2}^{\tilde{g}}(0)\right|$ for $M_{W} \lesssim \tilde{m}_{b}$. It should be remarked that, owing to the factor $1 / \cos \beta$, a large value for the ratio of the vacuum expectation values of the Higgs bosons proportionally enhances the chargino contribution.

Moreover, for the FCNC processes mediated by the supersymmetric particles to occur at sizable rates, the mass differences between the exchanged squarks have to be large, softening the cancellation between the different squark contributions which arises from the unitarity of the mixing matrix. The masses of the squarks in the first two generations are predicted to be almost degenerated, while if the squarks and the $t$-quark have the masses of the same order of magnitude, the masses of the $t$-squarks and $b$-squarks become different from other squarks'. These differences are much larger for the $t$-squarks than for the $b$ squarks because of a large mixing between the left-handed and right-handed $t$-squarks coming from the Yukawa couplings, as seen from eqs. (6) and (7). One of the $t$-squarks could become the lightest among the squarks. Hence the cancellation is not so severe for the process (a) compared to (c). On the other hand, the neutralinomediated process (b) does not make a large contribution since the interaction is not strong and the exchanged squarks are down-type.

We give the numerical results for the branching ratio (BR) of $B \rightarrow X_{s} \gamma$. In order to see each effect of the various SSM contributions, the evaluation is made assuming only one contribution instead of summing all. The BR due to the chargino contribution is shown in Fig. 1 for $\dot{m}_{2}=200 \mathrm{GeV}$
Table 1

The values of $\tilde{m_{Q}}$ and $\tan \beta$.

\begin{tabular}{lcccc}
\hline & (i) & (ii) & (iii) & (iv) \\
\hline$\tilde{m_{Q}}(\mathrm{GeV})$ & 200 & 200 & 300 & 300 \\
$\tan \beta$ & 2 & 10 & 2 & 10 \\
\hline
\end{tabular}

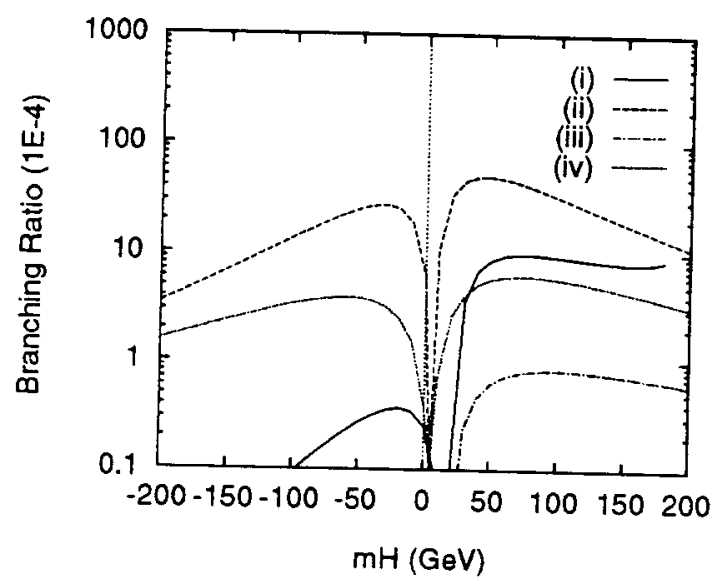

Figure 1. The inclusive branching ratio of $B \rightarrow$ $X_{s} \gamma$ due to the chargino contribution for the parameter sets in Table 1 with $\bar{m}_{2}=200 \mathrm{GeV}$.

and $-200 \mathrm{GeV} \leq m_{H} \leq 200 \mathrm{GeV}$. For $\tilde{m_{Q}}$ and $\tan \beta$ we take four sets of values listed in Table 1. The squark masses of the first two generations are given approximately by $\tilde{m_{Q}}$. The values of the lighter chargino mass and the lighter $t$-squark mass corresponding to these parameter values are shown in Fig. 2 and Fig. 3. For small values of $\left|m_{H}\right|$ the lighter chargino mass becomes less than $45 \mathrm{GeV}$, which is ruled out experimentally. Also the lighter $t$-squark should be heavier than $45 \mathrm{GeV}$. If the $t$-quark mass is around 150 $\mathrm{GeV}$, the BR due to the standard $W$-boson contribution with QCD corrections is $(3-5) \times 10^{-4}$. Compared to this value, the chargino contribution could give an equal or a larger amount of branching ratio if $\tan \beta$ is large and/or $\tilde{m_{t 1}}$ is small. The BR does not vary much with $\tilde{m}_{2}$ for $100 \mathrm{GeV} \lesssim \tilde{m}_{2} \lesssim 300 \mathrm{GeV}$. The sign of the 


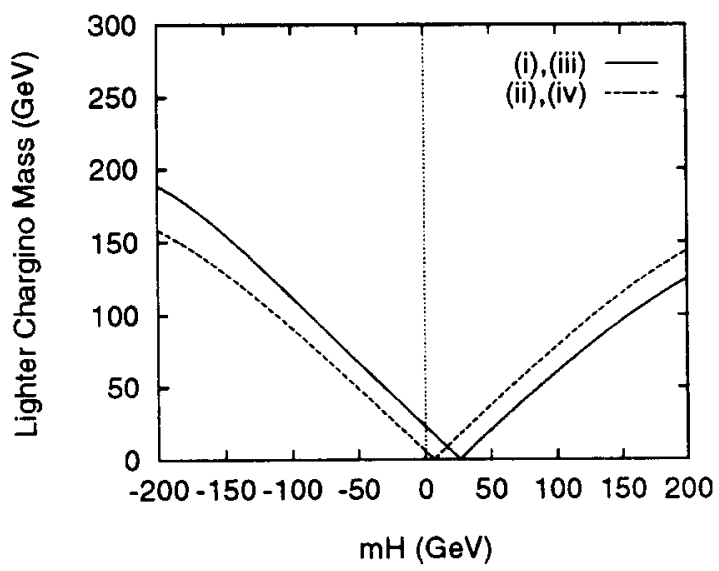

Figure 2. The mass of the lighter chargino for the $\tan \beta$ values in Table 1 with $\tilde{m}_{2}=200 \mathrm{GeV}$.

dipole form factor $F_{2}^{\tilde{\omega}}(0)$ depends on the parameters. Compared to the $W$-boson contribution $F_{2}^{W}(0)$ the sign is the same for $m_{H} \lesssim 0 \mathrm{GeV}$ and opposite for $m_{H} \gtrsim 50 \mathrm{GeV}$ in our examples.

In Fig. 4 we show the BR due to the gluino contribution for the three parameter sets (ii), (iii), and (iv) in Table 1 with $m_{H}=200 \mathrm{GeV}$ and $0 \mathrm{GeV} \leq \tilde{m}_{2} \leq 400 \mathrm{GeV}$. The gluino mass is determined through eq. (20). The curves are not shown in the ranges of small $\tilde{m}_{2}$ where the lighter chargino has a mass less than $45 \mathrm{GeV}$. For (i) one of the eigenvalues of the $t$-squark mass-squared matrix $M_{t}^{2}$ becomes negative, breaking $\mathrm{SU}(3)$. A large value of the $B R$ is only realized in (ii) for small values of $\tilde{m}_{2}$. The lighter $b$-squark mass is $157 \mathrm{GeV}$ in this example. The gluino contribution is negligible in other parameter regions. The sign of $F_{2}^{\tilde{g}}(0)$ depends on the parameters. In our examples the sign is opposite to $F_{2}^{W}(0)$. In Fig. 5 we show the BR due to the charged Higgs boson contribution for the values of $\tan \beta$ in Table 1 with $50 \mathrm{GeV} \leq M_{H \pm} \leq 400 \mathrm{GeV}$. As far as $\tan \beta>1$, the BR does not much depend on the value of $\tan \beta$. The sign of $F_{2}^{H \pm}(0)$ is the same as $F_{2}^{W}(0)$. The neutralino contribution is generally negligible. For further analyses from various points of view, see refs. $[4,5,6]$.

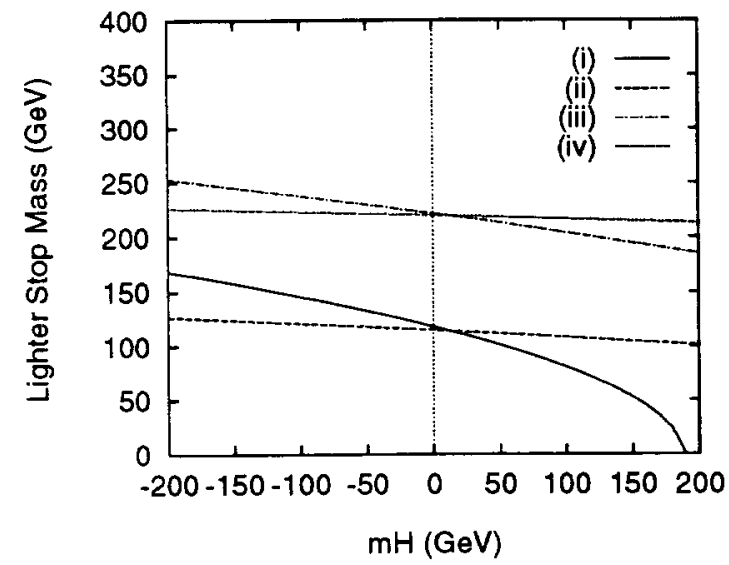

Figure 3. The mass of the lighter $t$-squark for the parameter sets in Table 1 with $\tilde{m_{2}}=200 \mathrm{GeV}$.

If some dipole form factors have values of the same order of magnitude, then their relative signs become important for predicting the net effect of the SSM. Although the interference between $F_{2}^{W}(0)$ and $F_{2}^{H \pm}(0)$ is constructive, both constructive and destructive interferences can happen for the ohter contributions.

\section{SUMMARY}

In the SSM there exist several new interactions which could induce FCNC processes. We have discussed their effects on the radiative decay $b \rightarrow s \gamma$. This decay can occur at the oneloop level through diagrams with the charginos, neutralinos, gluinos, or charged Higgs bosons, in addition to the standard diagrams with the $W$ bosons. Under the assumption of grand unification and $N=1$ supergravity the contribution of the chargino-loop diagrams dominates over other new contributions in wide rages of the parameter space. If $\tilde{m}_{2},\left|m_{H}\right|, \tilde{m_{Q}} \lesssim 300 \mathrm{GeV}$ and $\tan \beta \gtrsim 10$, the chargino contribution is likely larger than or equal to the contribution of the standard model. Compared with the standard model prediction, the branching ratio of $B \rightarrow$ $X_{s} \gamma$ becomes enhanced or, if the two contribu- 


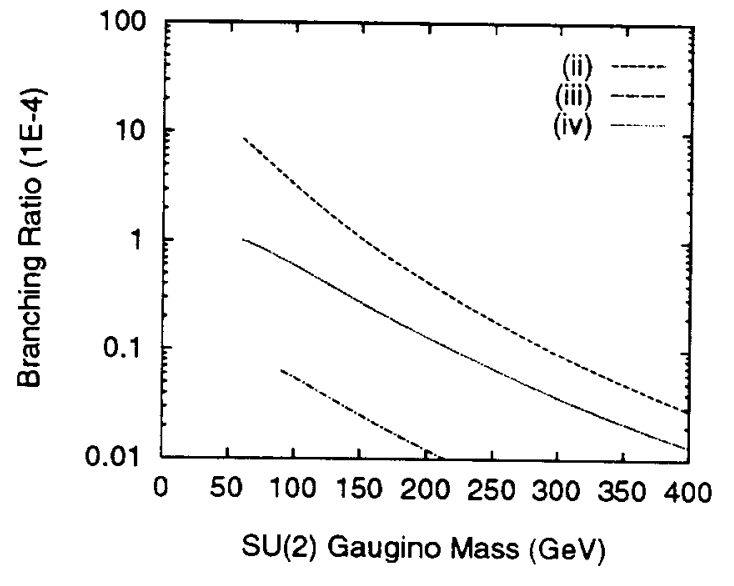

Figure 4. The inclusive branching ratio of $B \rightarrow$ $X_{s} \gamma$ due to the gluino contribution for the parameter sets in Table 1 with $m_{H}=200 \mathrm{GeV}$.

tions to the dipole form factor are not much different in magnitude and opposite in sign, reduced. Given the present experimental accomplishment for $b \rightarrow s \gamma$, detailed studies of the SSM effects taking into account all the possible contributions could give an useful constraint on the model.

\section{REFERENCES}

1. CLEO Collaboration, Phys. Rev. Lett. 71 (1993) 674.

2. S. Bertolini, F. Borzumati, and A. Masiero. Phys. Rev. Lett. 59 (1987) 180: N.G. Deshpande, P. Lo, J. Trampetic. G. Eilam, and P. Singer, Phys. Rev. Lett. 59 (1987) 183; B. Grinstein, R. Springer, and M.B. Wise, Phys. Lett. B202 (1988) 138.

3. P.J. O'Donnell, Phys. Lett. B175 (1986) 369: T. Altomari, Phys. Rev. D37 (1988) 677; N.G. Deshpande, P. Lo, and J. Trampetic, Z. Phys. C40 (1988) 369; C.A. Dominguez. N. Paver. and Riazuddin, Phys. Lett. B214 (1988) 459.

4. S. Bertolini. F. Borzumati, and A. Masiero. Phys. Lett. B192 (1987) 437; A. Masiero and G. Ridolfi, Phys. Lett. B212 (1988) 171; T.M. Aliev. A.A. Ovchinnikov, and V.A. Slobo-

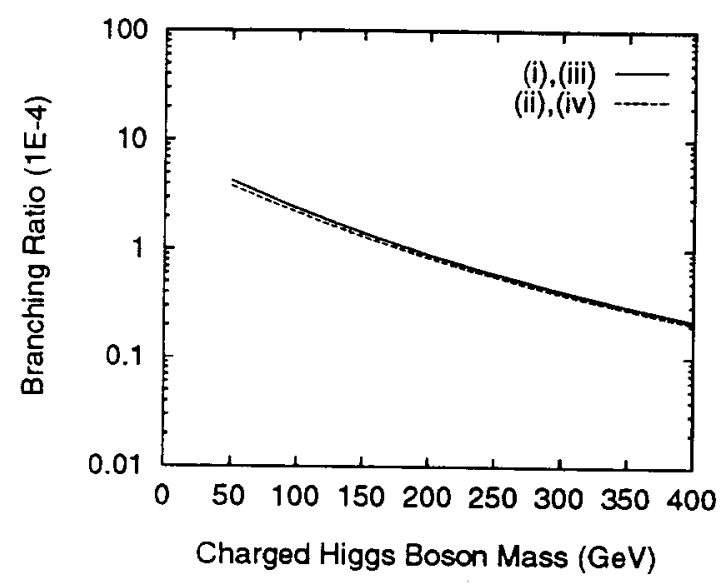

Figure 5. The inclusive branching ratio of $B \rightarrow$ $X_{s} \gamma$ due to the charged Higgs boson contribution for the $\tan \beta$ values in Table 1 .

denyuk, Phys. Lett. B237 (1990) 569; I.I. Bigi and F. Gabbiani, Nucl. Phys. B352 (1991) 309; S. Bertolini, F. Borzumati, A. Masiero, and G. Ridolfi, Nucl. Phys. B353 (1991) 591.

5. N. Oshimo, Nucl. Phys. B404 (1993) 20.

6. J.L. Hewett, Phys. Rev. Lett. 70 (1993) 1045; V. Barger, M.S. Berger, and R.J.N. Phillips, Phys. Rev. Lett. 70 (1993) 1368; J.L. Lopez, D.V. Nanopoulos, and G.T. Park, Phys. Rev. D48 (1993) R974; M.A. Díaz, Phys. Lett. B304 (1993) 278; B322 (1994) 207; R. Barbieri and G.F. Giudice, Phys. Lett. B309 (1993) 86; Y. Okada, Phys. Lett. B315 (1993) 119; R. Garisto and J.N. Ng, Phys. Lett. B315 (1993) 372; F.M. Borzumati, DESY-93-090.

7. B. Grinstein and M.B. Wise, Phys. Lett. B201 (1988) 274; W.-S. Hou and R.S. Willey, Phys. Lett. B202 (1988) 591; Nucl. Phys. B326 (1989) 54; B. Grinstein, M.J. Savage, and M.B. Wise, Nucl. Phys. B319 (1989) 271.

8. M.J. Duncan, Nucl. Phys. B221 (1983) 285; J.F. Donoghue, H.P. Nilles, and D. Wyler, Phys. Lett. 128B (1983) 55. 